\title{
Impact of injection sites for soluble insulin on glycaemic control in Type 1 (insulin-dependent) diabetic patients treated with a multiple insulin injection regimen
}

\author{
J.E.Henriksen ${ }^{1}$, M.S. Djurhuus ${ }^{2}$, A. Vaag ${ }^{1}$, P. Thye-Rønn ${ }^{1}$, D. Knudsen ${ }^{3}$, O. Hother-Nielsen ${ }^{1}$, H. Beck-Nielsen ${ }^{1}$ \\ ${ }^{1}$ Diabetes Research Centre, Odense University and Department of Endocrinology and Internal Medicine M, Odense University Hospital, \\ Odense, Denmark \\ ${ }^{2}$ Department of Clinical Chemistry, Odense University Hospital, Odense, Denmark \\ ${ }^{3}$ Department of Radiology, Odense University Hospital, Odense, Denmark
}

\begin{abstract}
Summary. The absorption rate of rapid acting (soluble) insulin is slow from the subcutaneous tissue of the thigh compared to intramuscular injection into the thigh and s.c. injection into the abdominal wall. The aim of the study was to evaluate the impact of soluble insulin injected either intramuscularly into the thigh (IMT), s. c. into the abdominal wall (SCA) or s.c. into the thigh (SCT) on glycaemic control in Type 1 (insulin-dependent) diabetic outpatients treated with the basal bolus insulin delivery regimen. Fifty-five, C-peptide negative Type 1 diabetic outpatients were included in a randomised 3-month intervention study. The insulin doses were adjusted frequently by blinded observers based on the patients' self-monitored blood glucose values and reported hypoglycaemic episodes. The serum fructosamine value was within normal limits in three patients in the IMT group, in six patients in the SCA group and in none of the patients in the SCT group following the intervention period $(p<0.01)$. However, the difference in mean serum fructosamine values did not reach statistical significance (IMT: $1.24 \mathrm{mmol} / \mathrm{l}(95 \%$ confidence interval; 1.17 to 1.31 ), SCA: $1.25 \mathrm{mmol} / \mathrm{l}$ (1.18 to
\end{abstract}

$1.32)$, SCT: $1.34 \mathrm{mmol} / \mathrm{l}(1.26$ to 1.41$),(p=0.09))$. Blood glucose excursions were larger in the SCT group than in the SCA and IMT group from post-lunch to pre-dinner measurements and from pre- to post-dinner measurements. A higher number of measured low nocturnal blood glucose values (less than $4 \mathrm{mmol} / \mathrm{l}$ ) was observed in the SCT group (34 of 85) than in the IMT (14 of 64) and SCA (21 of 81) group $(p<0.05)$. Three patients in the IMT group, two in the SCA group, and seven in the SCT group experienced severe hypoglycaemic episodes $(p=0.14)$. In conclusion s. c. injection of soluble insulin into the abdominal wall is preferable compared to s. c. injection into the thigh in the basal bolus insulin delivery regimen. Furthermore, soluble insulin injection s.c. into the thigh during daytime has important clinical implications for the development of nocturnal hypoglycaemia independently of the NPH insulin injection at bedtime.

Key words: Insulin pharmacokinetics, intramuscular insulin injection, subcutaneous insulin injection, blood glucose control, nocturnal hypoglycaemia.
The multiple insulin injection regimen (basal bolus insulin delivery regimen) is based on injections of soluble (rapid acting) insulin at mealtimes and injections of NPH (intermediate acting) insulin at bedtime. The purpose of this insulin regimen is to mimic the normal diurnal plasma insulin profile [1]. Thus, the purpose of the bolus component is to provide insulin delivery adequately timed to the absorption of the meal, whereas the purpose of the basal component is to provide adequate insulinisation during the night time and between meals. Obviously, insulin delivery depends on the absorption rate of the injected insulin and this has been demonstrated to vary considerably between different anatomical regions [212].

It has been shown, that the preferred injection site for $\mathrm{NPH}$ insulin is the subcutaneous (s.c.) tissue of the thigh $[8,10-12]$. Thus, this injection site produces the most con- stant insulin absorption rate with the smallest peak in plasma insulin [8, 10-12]. Furthermore, the subcutaneous tissue of the thigh is the injection site with the lowest intrapatient coefficient of variation of absorption [10].

However, the absorption rate of soluble insulin from the subcutaneous tissue of the thigh is too slow to adequately mimic the normal spikes in plasma insulin following the meals [2]. Thus, approximately $50 \%$ of the s.c. injected insulin is absorbed after $5 \mathrm{~h}$ compared to $2 \mathrm{~h}$ after intramuscular (i.m.) injection into the thigh [2,4]. The absorption rate of soluble insulin from the abdominal wall is as fast as i.m. injected insulin into the thigh $[4,8,13]$ and the absorption rate is faster than seen after s. c. injection into the thigh [6-8]. The consequence of a slow absorption rate of soluble insulin is relative hypoinsulinaemia after a meal and relative hyperinsulinaemia before the next meal. In turn, this enhances post-prandial hyperglycaemia 
Table 1. Clinical characteristics, at randomisation, of 49 Type 1 diabetic patients randomly assigned for use of either the intramuscular tissue of the thigh (IMT), the subcutaneous tissue of the abdominal wall (SCA) or the subcutaneous tissue of the thigh (SCT) for injection of soluble insulin

\begin{tabular}{llll}
\hline Group & IMT & SCA & SCT \\
\hline$n$ & 14 & 17 & 18 \\
Sex (male/female) & $9 / 5$ & $10 / 7$ & $12 / 6$ \\
Age (years) & $30.4(18-48)$ & $33.8(18-56)$ & $34.7(18-57)$ \\
Duration of diabetes (years) & $12.6(5-22)$ & $16.5(2-29)$ & $14.4(1-28)$ \\
Number of patients with retinopathy & & 3 & 7 \\
$\quad$ non-proliferative & 4 & 2 & 0 \\
$\quad$ proliferative & 1 & 5 & 0 \\
Number of patients with microalbuminuria & 4 & $23.8(22.7$ to 24.9$)$ & $24.6(23.3$ to 24.9$)$ \\
BMI (kg/m $\left.{ }^{2}\right)$ & $23.4(22.0$ to 24.7$)$ & $70.5(65.7$ to 75.4$)$ & $74.5(69.2$ to 79.9$)$ \\
Weight (kg) & $71.9(66.4$ to 77.4$)$ & $8.3(7.6$ to 9.1$)$ & $8.7(8.0$ to 9.3$)$ \\
Glycated haemoglobin (\%) & $8.1(7.4$ to 8.8$)$ & $1.39(1.29$ to 1.48$)$ & $1.48(1.37$ to 1.59$)$ \\
Serum fructosamine (mmol/l) & $1.40(1.29$ to 1.51$)$ & & 2 \\
Patients with se-fructosamine value within normal & & 1 & $11 \%(1$ to 35$)$ \\
range & & $6 \%(0$ to 29$)$ & \\
number of patient & 0 & & \\
\% (95\% CI) & $0 \%(0$ to 23) & & \\
\hline
\end{tabular}

Values are mean (range or $95 \%$ confidence interval)

and causes an increased risk for pre-prandial hypoglycaemia.

We previously demonstrated in an inpatient cross-over study that i.m. injection of soluble insulin into the thigh clearly generates an insulin profile with meal-related peaks in plasma insulin whereas the peaks obtained after s. c. injection into the thigh were blunted [2]. Consequent$\mathrm{ly}$, the coefficient of variation of blood glucose concentrations during the study period was significantly higher following s.c. injections into the thigh compared to the period with i.m. injections. Therefore, our previous study indicated that i.m. injection of soluble insulin in the basal bolus insulin delivery regimen may be beneficial compared to s. c. injection into the thigh. On the other hand, other investigators have warned against an increased risk for exercise-induced hypoglycaemia following i.m. injection of soluble insulin $[3,14]$. Therefore, studies have been requested [14] to evaluate the impact of insulin injection site for long-term metabolic control, risk for hypoglycaemic episodes and acceptability to patients.

The present study was designed to answer these questions in a single blinded 3-month outpatient setting, where patients were randomly assigned to injection of soluble insulin into either the intramuscular tissue of the thigh (IMT), the subcutaneous tissue of the abdominal wall (SCA) or the subcutaneous tissue of thigh (SCT). NPH insulin was injected s. c. into the thigh in all patients.

\section{Patients and methods}

\section{Patients}

Type 1 diabetic patients, attending the outpatient clinic at Odense University Hospital, Department of Endocrinology, were invited to participate in the study. Fifty-five patients gave informed consent and were included. All patients were without endogenous insulin secretion (C-peptide $<0.06 \mathrm{nmol} / \mathrm{l}$ ). Patients were treated with soluble insulin (Actrapid HM or Velosulin HM; Novo-Nordisk, Bagsvaerd, Denmark) before breakfast, lunch and dinner and intermediate acting insulin (Protaphane HM or Insulatard HM; Novo-Nordisk) at bedtime. Six patients were later excluded from the study due to insufficient compliance (i.e. performed less than $25 \%$ of requested blood glucose measurements). Three patients were from the IMT group, two from the SCA group and one from the SCT group. Clinical characteristics are given in Table 1.

All patients were carefully informed about the nature and purpose of the study before their consent to participate was obtained. The protocol of the study was reviewed and approved by the regional ethical committee.

\section{Protocol and methods}

After a 1 month run-in period patients were randomized to three different injection sites for soluble insulin for 3 months using either i. m. injection into the thigh (IMT), s.c. injection into the abdominal wall (SCA) or s.c. injection into the thigh (SCT). In all three groups intermediate acting insulin at bedtime was taken s.c. into the thigh. Patients visited the outpatient clinic at the start of the run-in period, at randomisation and $1-, 2.5$ - and 3 months after randomisation. In addition to the scheduled visits to the outpatient clinic frequent contacts were made to the patients by telephone and letter to achieve or improve compliance. At the end of the study patients were asked to answer a questionnaire regarding the injection site and technique they had used before and during the study. The patients were also asked about their physical activity level during the study period.

Subcutaneous thickness and injection technique. In patients randomized to thigh injection for soluble insulin the thickness of the subcutaneous tissue was determined with an ultrasound scanner (Picker 9500; Hitachi, Tokyo, Japan) using a linear transducer (5 MHZ R40). Thickness of the subcutaneous tissue was measured at nine different sites in the anterolateral area on each thigh and noted in a diagram. Using this diagram patients in the SCT and IMT groups were instructed to use appropriate areas of the thigh to ensure either 
Table 2. Metabolic control at the end of intervention, severe hypoglycaemic episodes during the intervention period and mild hypoglycaemic episodes in the last 14 days period of the study in 49 Type 1 diabetic patients who used different injection sites for soluble insulin (IMT, intramuscular tissue of the thigh; SCA, subcutaneous tissue of the abdominal wall; SCT, subcutaneous tissue of the thigh)

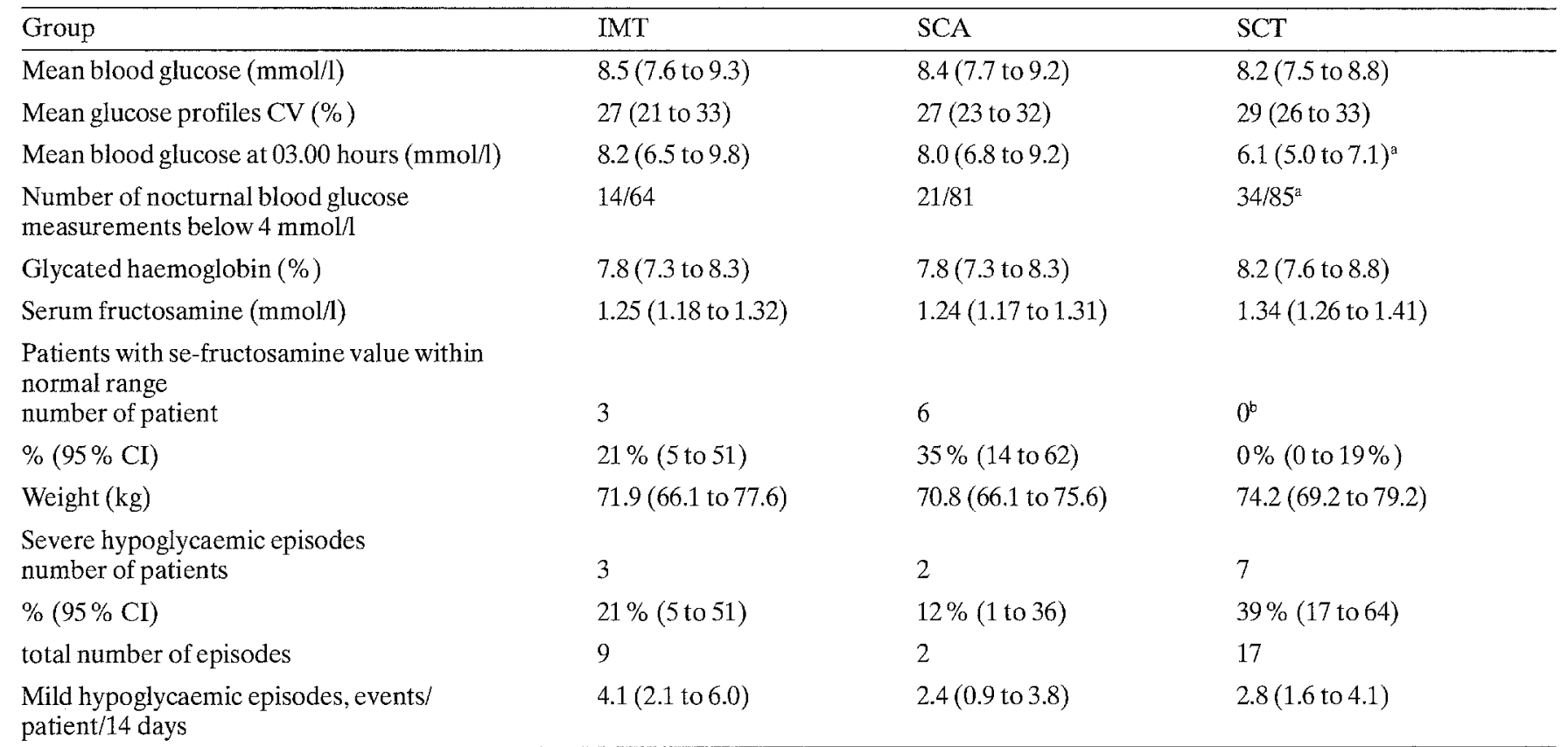

${ }^{\mathrm{a}} p<0.05,{ }^{\mathrm{b}} p<0.01$, vs IMT, SCA by one-way ANOVA. Values are mean and $95 \%$ confidence interval or proportion and $95 \%$ confidence interval. CV, Coefficient of variation of the mean daily blood glucose profile

true s.c. or true i.m. placement of insulin. Mean thickness of the subcutaneous tissue was $10.6 \mathrm{~mm}$ (range 5.5 to 18.4) and was larger in the medial and proximal part of the thigh than in the lateral and distal part of the thigh $(11.9 \pm 0.6 \mathrm{~mm}$ vs $8.8 \pm 0.5 \mathrm{~mm})$. None of the patients had any lipodystrophy at the injection sites either at the thigh or at the abdomen.

The SCA group was instructed to use the area from $10 \mathrm{~cm}$ above to $10 \mathrm{~cm}$ below the umbilicus and up to $15 \mathrm{~cm}$ lateral to each side.

For s. c. injections $12.5 \mathrm{~mm}$ needles were used and injection given into an elevated skin fold at an angle of $45^{\circ}$. For i.m. injection $16 \mathrm{~mm}$ needles were used with perpendicular injection without elevation of the skin.

The patients injected soluble insulin immediately after blood glucose measurements before meals. NPH insulin was injected at 22.00 hours. Intermediate acting insulin was injected s.c. into the thigh in all three groups.

Blood samples. Three times during the study period (at run-in, randomisation and at the end of the study) blood was sampled for measurements of $\mathrm{HbA}_{1 \mathrm{c}}$ (HPLC, normal range 5.4 to $7.4 \%$ ), and serum (se) fructosamine (corrected for variation in se-albumin (normal range 0.86 to $1.14 \mathrm{mmol} / 1[15,16])$.

Equipment. At the start of the run-in period each patient received a glycometer (Reflolux S; Bochringer Mannheim GmbH Diagnostica, Mannheim, FRG) together with a connectable memory device (CAMIT EL; Boehringer Mannheim GmbH Diagnostica). All glycometers were tested and certified as error-free by a specially trained technician. Test strips (Haemoglycotest 1-44R) were all from the same batch. Patients were carefully instructed in both theory and practice of the use of the glycometer and memory device.

Blood glucose measurement. During the first month of the intervention period blood glucose was measured daily four times per day i.e. before meals and at bedtime. After that blood glucose was measured twice per week with measurements before and $1.5 \mathrm{~h}$ after meals and at bedtime. During the last 2 weeks of the study period blood glucose was measured before and after meals, at bedtime and at 03.00 hours.
The latter measurements were used in the comparison of the three different injection sites.

Insulin dose adjustment. During the adjustment period (0-2.5 months) insulin doses were adjusted by trained diabetologists who were unaware of the injection site on each individual. Thus adjustments were made each week during the first month and after that every second week. Adjustments were made based on blood glucose profiles and reported hypoglycaemic episodes. The aim of these adjustments was to achieve "near normal" pre-meal blood glucose values. Thus, the target was $4-7 \mathrm{mmol} /$ for pre-meal blood glucose values and $7-10 \mathrm{mmol} / \mathrm{l}$ for glucose values measured $1.5 \mathrm{~h}$ after meals and at bedtime.

Hypoglycaemia. Two categories of hypoglycaemic episodes were defined in the protocol. Mild hypoglycaemic attacks were defined as episodes which the patient could handle himself by intake of glucose. If the patient became dependent on help from other persons the attack was defined as "severe". Only three mild hypoglycaemic episodes were allowed per week. For higher frequencies insulin doses were reduced or redistributed.

\section{Statistical analysis}

Differences between values before and after the intervention period were evaluated using Student's $t$-test for paired data. Differences between groups were determined by one-way analysis of variance. Differences in distributions between the groups were assessed with the G-test [17].

For each patient a mean diurnal blood glucose profile was calculated from the final 6 days of the study. The coefficient of variation (CV) and changes in blood glucose values during the day were thereafter calculated from this profile. Differences between the groups in $\mathrm{CV}$, in blood glucose concentrations and in changes in blood glucose concentrations were then compared using one-way ANOVA. Data are presented as mean and $95 \%$ confidence limits. Differences with $p$-values less than 0.05 were considered significant. 
Table 3. Total daily insulin dose, soluble insulin dose at mealtime and NPH insulin dose at bedtime in 49 Type 1 diabetic patients after 3 months of intensified insulin treatment and difference between insulin doses at randomisation and after 3 months. Soluble insulin was injected: IMT, i. m. into the thigh; SCA, s.c. into the abdominal wall; SCT. s. c. into the thigh

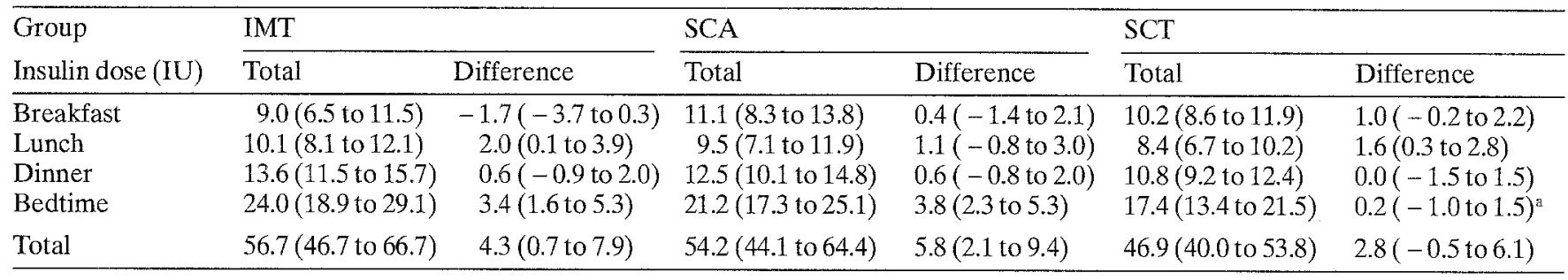

${ }^{a} p<0.001$ vs IMT, SCA by one-way ANOVA. Values are mean and $95 \%$ confidence interval

\section{Results}

\section{Overall metabolic control}

Metabolic control was similar in the three groups at the start of the study and remained unchanged during the runin period (Table 1).

Following the intervention period both $\mathrm{HbA}_{1 \mathrm{c}}$ value (8.39\% (95\% CI, 8.00 to 8.78$)$ to $7.94 \%$ (7.64 to 8.24$)$, $(p<0.002))$ and se-fructosamine value $(1.42 \mathrm{mmol} / \mathrm{l}(1.37$ to 1.48$)$ to $1.27 \mathrm{mmol} / 1(1.23$ to 1.32$),(p<0.0001))$ were reduced compared to before the study.

There was no difference between the groups in $\mathrm{HbA}_{10}$ value or in se-fructosamine value at the end of the study although there was a tendency towards a higher se-fructosamine value in the SCT group (Table 2, $p=0.09$ ). However, following intervention se-fructosamine values were normal in three patients in the IMT group, in six patients in the SCA group and in none of the patients in the SCT group (Table 2). This was in contrast to before intervention where se-fructosamine values were normal in none of the patients in the IMT group, one patient in the SCA group and two patients in the SCT group (Table 1).

\section{Glycaemic control during the last 2 weeks}

Figure 1 shows the mean diurnal blood glucose values for the three groups. The profiles for the IMT and the SCA groups look similar, whereas the profile for the SCT group has another appearance with larger fluctuations in blood glucose values at the end of the day, although there was no difference in the CV (Table 2, NS). However, blood glucose concentrations from post-lunch measurement to predinner measurement decreased $1.2 \mathrm{mmol} / \mathrm{l}(-2.6$ to 0.3$)$ in the SCT group compared to an increase in the IMT and the SCA groups $(0.9 \mathrm{mmol} / \mathrm{l}(-0.8$ to 2.5$)$ and $1.0 \mathrm{mmol} / \mathrm{l}$ $(0.0$ to 2.0$),(p<0.04))$. Also the increase in blood glucose concentration from pre- to post-dinner measurements was higher in the SCT group than in the SCA and IMT group (IMT: $1.5 \mathrm{mmol} / \mathrm{l}(0.4$ to 2.6$), \mathrm{SCA}: 1.1 \mathrm{mmol} / \mathrm{l}(-0.1$ to 2.3$)$, SCT: $2.7 \mathrm{mmol} / \mathrm{l}(2.0$ to 3.5$),(p<0.05))$. Blood glucose concentration from bedtime to 03.00 hours decreased $2.9 \mathrm{mmol} / \mathrm{l}(1.6$ to 4.3$)$ in the SCT group compared to only $0.5 \mathrm{mmol} / 1(-0.6$ to 1.5$)$ in the IMT group and $0.1 \mathrm{mmol} / 1$ $(-1.7$ to 1.8$)$ in the SCA group $(p<0.008)$. All other changes in blood glucose concentrations during the day were similar between the groups.
Mean blood glucose value at night was significantly lower in the SCT group than in the other two groups (Fig. 1 and Table 2). This was due to a statistically significant larger number of measured blood glucose values below $4 \mathrm{mmol} / \mathrm{l}$ in the SCT group (Fig. 1 and Table 2).

\section{Hypoglycaemia}

There was a tendency towards a higher proportion of patients with severe hypoglycaemic episodes in the SCT group (Table $2, p=0.16$ ) and a tendency towards more episodes (Table 2, Kruskal-Wallis one-way ANOVA, $p=0.14$ ). Seven hypoglycaemic episodes were with loss of consciousness. One patient in the IMT group experienced two episodes, three patients in the SCT experienced four episodes and one patient in the SCA group experienced one episode.

\section{Insulin dose}

Total daily insulin dose was increased in all but the SCT group during the intervention period (Table 3). However, the difference between the groups did not attain statistical significance. On the other hand, a clear difference between the groups was noted in the bedtime NPH insulin dose (Table 3). Thus, in the SCA and IMT groups the NPH insulin dose increased whereas in the SCT group there was no increase (Table 3 ). Despite the increase in insulin dose, body weight remained unchanged (Table 2).

\section{Physical activity level and acceptability of injection site}

There was no difference in the level of physical activity between the groups either in the level during the working hours (total hours per week of walking and of moderate to hard physical activity: IMT: 19.0 (9.5 to 28.5), SCA: 18.3 (9.8 to 26.7$)$, SCT: 21.8 (10.6 to 33.0 ), (NS)) or during the leisure time (total hours per week of biking, jogging, running or other forms of sport: IMT: $5.3(0.3$ to 10.4), SCA: 7.9 (4.1 to 11.6), SCT: 4.3 (2.3 to 6.3), (NS)).

Of the patients who injected soluble insulin into the abdominal wall before the study and who were randomly assigned to the SCT group (16 patients), 7 patients claimed that they had equal pain at the new injection site, 7 pa- 


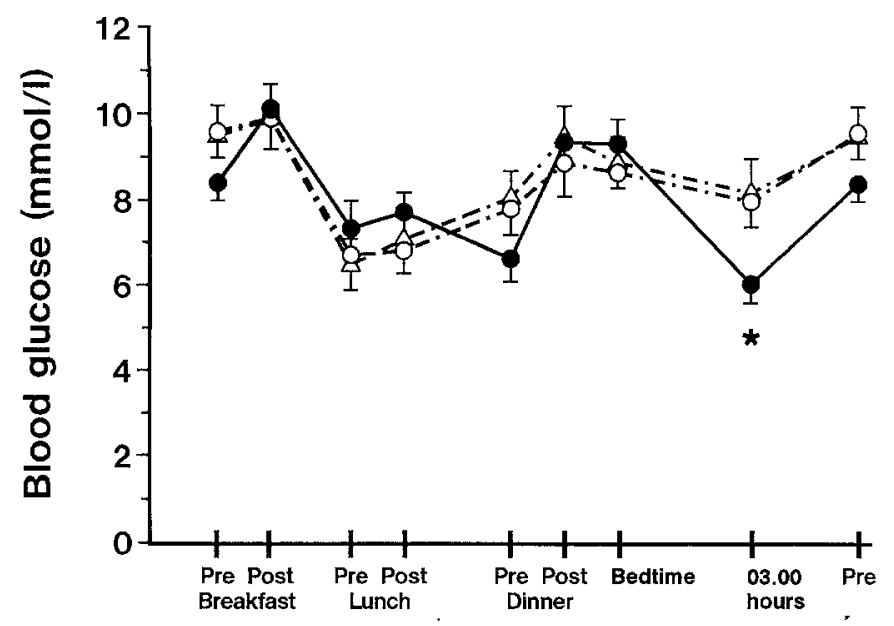

Fig. 1. Diurnal blood glucose values in three groups of Type 1 diabetic patients randomized to injection of soluble insulin either i.m. into the thigh $(\Delta)$, s. c. into the abdominal wall $(O)$ or s. c. into the thigh ( ) at mealtimes. Intermediate acting insulin at bedtime was taken s.c. into the thigh in all patients. Values represent mean ( \pm SEM) from six blood glucose profiles (eight measurements per day) per patient measured during the last 2 -week period of the 3 -month intervention period. ${ }^{*} p<0.03$

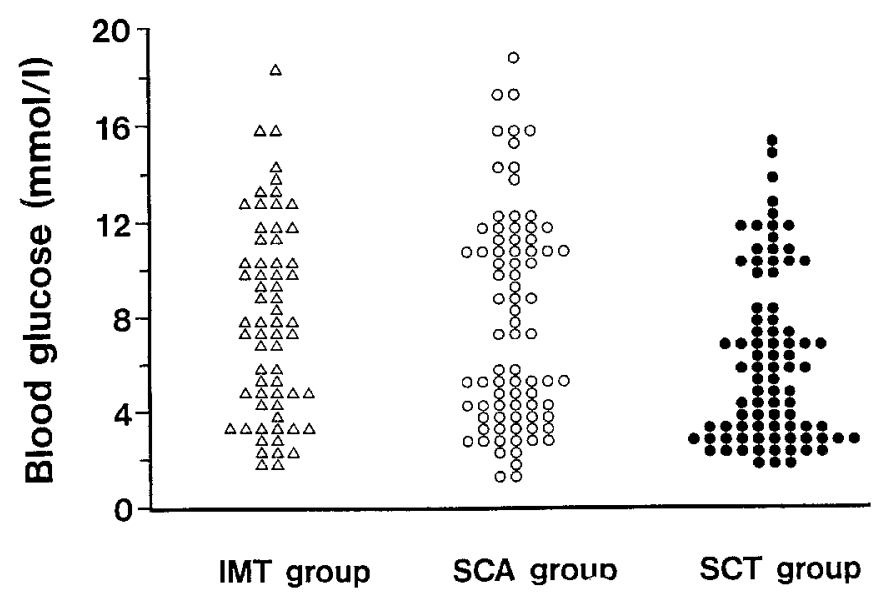

Fig. 2. Nocturnal blood glucose values measured during the last 2 weeks of the 3 -month intervention period in three groups of Type 1 diabetic patients randomized to injection of soluble insulin either i.m. into the thigh (IMT), s.c. into the abdominal wall (SCA) or s.c. in to the thigh (SCT) at mealtimes. The SCT group had a higher number of blood glucose values below $4 \mathrm{mmol} / \mathrm{l}(p<0.05)$

tients had greater pain and 2 had less pain. In the IMT group (13 patients) 7 had equal pain, 5 had more pain and 1 had less pain. The patients were also asked if they would use the injection site they had used in the study in the future if we could recommend it. All patients who used the SCA would accept it and 9 of 14 patients in the IMT group and 13 of 18 patients in the SCT group would accept the used injection site. Given a free choice most patients preferred the abdominal wall as the site for soluble insulin injections. Thus, 36 patients would prefer the abdominal wall, 2 patients the subcutaneous tissue of the thigh, 3 patients the intramuscular tissue of the thigh and 8 patients would prefer to alternate between injection sites $(p<0.001)$.
J.E.Henriksen et al.: Soluble insulin injection and glycaemic control

\section{Discussion}

The present study demonstrates that soluble insulin injection into the subcutaneous tissue of the abdominal wall and into the intramuscular tissue of the thigh produces equal glycaemic control, whereas use of the subcutaneous tissue of the thigh is followed by larger blood glucose excursions including a higher risk of nocturnal hypoglycaemia.

In our outpatient clinic we aim for patients to have $\mathrm{HbA}_{1 \mathrm{c}}$ values below $8.0 \%$. At the end of the 3-month intensified insulin treatment period, the IMT and SCA group had mean values below this value but not the SCT group. However, the $\mathrm{HbA}_{1 \mathrm{c}}$ value was not different between the groups. $\mathrm{HbA}_{1 \mathrm{c}}$ and se-fructosamine are assumed to reflect glycaemic control during the preceding 2-3 months and 4-6 weeks, respectively [18] and in this way se-fructosamine can be assumed to reflect glycaemic control more precisely at the end of intervention. There was a tendency towards a lower se-fructosamine value in the SCA and IMT groups, although this did not reach statistical significance. However, more patients in the IMT and SCA groups than in the SCT group had a normal sefructosamine value following intervention.

Blood glucose concentrations increased more after the meal and decreased more after the postprandial blood glucose peak around lunch and dinner in the SCT group. This is in agreement with our previous findings [2]. Due to the slow absorption rate of soluble insulin from the subcutaneous tissue of the thigh, blood glucose concentrations would increase more after meals and due to the prolonged action of the injected insulin blood glucose concentrations would decline more from the postprandial blood glucose peak to the next meal. Thus, in our previous inpatient study we found a significantly lower CV of diurnal blood glucose concentrations after i.m. injection of soluble insulin into the thigh than after s.c. injection into the thigh [2].

An important finding in the present study was the marked decrease in blood glucose concentrations from bedtime to 03.00 hours in the SCT group. The lower nocturnal blood glucose concentration in the SCT group was due to a higher incidence of blood glucose values below $4 \mathrm{mmol} / \mathrm{l}$. Nocturnal hypoglycaemia is a major problem in the treatment of the diabetic patient [19-25]; several risk factors have been identified [23-25]. The present study indicates a formerly unknown risk factor. An increased risk for nocturnal hypoglycaemia was demonstrated when soluble insulin was injected s.c. into the thigh during the day.

It is important to realise that the increased number of nocturnal low blood glucose values in the SCT group was not due to an increased NPH insulin dose at bedtime. In fact, the bedtime NPH insulin dose remained unchanged in the SCT group, whereas the NPH insulin dose in the SCA and IMT group was increased by about 4 units during the intervention period by the single blinded diabetes treatment team. Therefore, if the NPH insulin dose in the SCT group had been increased as in the SCA and IMT group it seems reasonable to assume an even higher number of low blood glucose values at night in the SCT group. 
Another risk factor for nocturnal hypoglycaemia is blood glucose concentration at bedtime, thus the lower the blood glucose concentration the higher the risk for developing nocturnal hypoglycaemia [23-25]. However, blood glucose concentration at bedtime was similar in the three groups. Also, the injection site for NPH insulin at bedtime was the same in the three groups. It thus seems unlikely that differences in the absorption rate of NPH insulin between the groups could have been responsible for the higher number of low blood glucose values in the SCT group.

The finding that the injection site for soluble insulin during the day markedly influences the blood glucose concentrations at night has important clinical implications. Thus, a low blood glucose concentration at night is usually prevented by a reduction of the bedtime NPHinsulin dose [26]. However, this study demonstrates that the insulin injection site and, thereby also the insulin doses of soluble insulin during the day has a great influence on the nighttime blood glucose concentrations. This means that the day time insulin injection technique and the insulin dose should be considered in the future if a low nocturnal blood glucose concentration is inadequately controlled by a reduction of the NPH insulin dose. In this context it should be noted that the pre-dinner soluble insulin injection in particular may be the prime candidate to influence nighttime blood glucose concentration.

It has been suggested that i.m. injection of soluble insulin might be dangerous due to an elevated risk of exercise-induced hypoglycaemic episodes and for this reason that i.m. injection should be avoided [4]. During our 3month study there was no evidence of such an elevated risk for severe hypoglycaemic episodes in the IMT group. In contrast, more patients in SCT group experienced severe hypoglycaemic episodes although this did not attain statistical significance. The difference could not be explained by a difference in the level of physical activity as this was comparable between the groups. Severe hypoglycaemic attacks in the SCT group could be due to the delayed insulin absorption from the subcutaneous tissue of the thigh resulting in inappropriate hyperinsulinaemia hours after the meal. This, together with exercise could be potentially dangerous regarding the development of severe hypoglycaemic episodes.

If a patient refuses to use the abdominal wall for injection of soluble insulin he should be advised to use the intramuscular tissue of the thigh. Among our patients the acceptability of this injection site was as high as the acceptability of the s.c. injection site of the thigh. Also the pain after i. m. injection was comparable to that seen after s.c. injection into the thigh. This is consistent with the findings of Wynne et al. [27], who have demonstrated that i.m. injection of insulin with a thin needle is no more painful than s.c. injection. Importantly, the thickness of the subcutaneous tissue is thinner on the lateral and distal aspects of the thigh. Therefore, this is the most appropriate area to recommend, if it is not possible to assess the precise subcutaneous thickness using ultrasound measurement. Furthermore, a $16 \mathrm{~mm}$ long needle should be used with injection given perpendicular to the skin.

However, most of our patients preferred the abdominal wall as injection site for soluble insulin. Obviously, this in- jection site is more convenient at mealtime than the injection sites of the thigh. The abdominal wall is often easily accessible also in public places whereas thigh injection most often must be preceded by removal of more clothing. On the other hand, from a pharmacokinetic point of view the best injection site for NPH insulin is the thigh and also from a practical point of view thigh injection at bedtime is acceptable.

In conclusion we have demonstrated that an equal glycaemic control can be obtained with i.m. injection of soluble insulin into the thigh as with s.c. injection into the abdominal wall and that i.m. injection is not associated with an increased risk of severe hypoglycaemia. Injection of soluble insulin into the thigh s.c. at mealtime is followed by a non-favourable glycaemic control with larger blood glucose excursions and an enhanced risk for nocturnal hypoglycaemia. We therefore recommend that soluble insulin in the basal bolus insulin regimen should be injected s.c. into the abdominal wall or alternatively i.m. into the thigh, whereas s.c. injection of soluble insulin into the thigh should be avoided.

Acknowledgements. We acknowledge the financial support from Novo Nordisk Farmaka Denmark and Boehringer Mannheim, Mannheim, FRG for supplying the patients with Reflolux $S$ and CAMITEL. We also would like to thank Mr. I. Johansen for measuring fructosamine and Mr. B.Pedersen for testing the glycometers prior to the study.

\section{References}

1. Rizza RA, Gerich JE, Haymond MW et al. (1980) Control of blood sugar in insulin-dependent diabetes: comparison of an artificial endocrine pancreas, continuous subcutaneous insulin infusion, and intensified conventional insulin therapy. $\mathrm{N}$ Engl $\mathbf{J}$ Med 303: 1313-1318

2. Vaag A, Damgaard Pedersen K, Lauritzen $\mathbf{M}$, Hildebrandt $\mathbf{P}$, Beck-Nielsen H (1990) Intramuscular versus subcutaneous injection of unmodified insulin: consequences for blood glucose control in patients with type 1 diabetes mellitus. Diabetic Med 7: 335-342

3. Frid A, Östman J, Linde B (1990) Hypoglycemia risk during exercise after intramuscular injection of insulin in thigh in IDDM. Diabetes Care 13: 473-477

4. Frid A, Gunnarsson R, Güntner P, Linde B (1988) Effects of accidental intramuscular injection on insulin absorption in IDDM. Diabetes Care 11: 41-45

5. Binder C (1969) Absorption of injected insulin: a clinical-pharmacological study. Acta Pharmacol Toxicol 27 [Suppl 2]: 1--87

6. Koivisto VA, Felig P (1980) Alterations in insulin absorption and in blood glucose control associated with varying insulin injection sites in diabetic patients. Ann Intern Med 92: 59-61

7. Süsstrunk H, Morell B, Ziegler WH, Froesch ER (1982) Insulin absorption from the abdomen and the thigh in healthy subjects during rest and exercise: blood glucose, plasma insulin, growth hormone, adrenaline and noradrenaline levels. Diabetologia 22: 171-174

8. Henriksen JE, Vaag A, Ramsgaard Hansen I, Lauritzen M, Beck-Nielsen H (1990) Absorption of soluble insulin and NPH insulin from different injection sites. Diabetologia 33 [Suppl]: 60 (Abstract)

9. Spraul M, Chantelau E, Koumoulidou J, Berger M (1988) Subcutaneous or nonsubcutaneous injection of insulin. Diabetes Care 11: 733-736 
10. Vaag A, Handberg A, Lauritzen M, Henriksen JE, Damgaard Pedersen K, Beck-Nielsen H (1990) Variation in absorption of NPH insulin due to intramuscular injection. Diabetes Care 13: $74-76$

11. Henriksen JE, Vaag A, Ramsgaard Hansen I, Lauritzen M, Djurhuus MS, Beck-Nielsen H (1991) Absorption of NPH (isophane) insulin in resting diabetic patients: evidence for the subcutaneous injection in the thigh as the preferred site. Diabetic Med 8: 453457

12. Thow JC, Johnson AB, Fulcher G, Home PD (1990) Different absorption of isophane (NPH) insulin from subcutaneous and intramuscular sites suggests a need to reassess recommended insulin injection technique. Diabetic Med 7: 600-602

13. Owens DR, Jones MK, Hayes TM et al (1981) Comparative study of subcutaneous, intramuscular, and intravenous administration of human insulin. Lancet II: $118-122$

14. Thow J, Home P (1990) Insulin injection technique. Depth of injection is important. BMJ 301:3-4

15. Johson RN, Metcalf PA, Baker JR (1983) Fructosamine: a new approach to the estimation of serum glycosylprotein. An index of diabetic control. Clin Chim Acta 127: 87-95

16. Johansen I, Milling-Eriksen R, Tougaard L (1986) Albumin-dependent correction of S-Fructosamine (S-FAMN) in a reference population. Scand J Clin Lab Invest 46 [Suppl 185]: 141 (Abstract)

17. Sokal RR, Rohlf FJ (1969) Biometry. The principles and practice of statistics in biological research. Freeman, San Francisco, pp 599-601

18. Service FJ, O'Brien PC, Rizza RA (1987) Measurements of glucose control. Diabetes Care 10: 225-237

19. Pramming S, Thorsteinsson B, Bendtson I, Rønn B, Binder C (1985) Nocturnal hypoglycaemia in patients receiving conventional treatment with insulin. BMJ 291:376-379
20. Gale EAM, Tattersall RB (1979) Unrecognised nocturnal hypoglycaemia in insulin-treated diabetics. Lancet I: 1049m-1052

21. Anonymous (1987) Nocturnal hypoglycaemia in childhood diabetes. Lancet II: $253-254$

22. Macfarlane PI, Walters M, Stutchfield P, Smith CS (1989) A prospective study of symptomatic hypoglycaemia in childhood diabetes. Diabetic Med 6: 627-630

23. Schiffrin A, Suissa S (1987) Predicting nocturnal hypoglycemia in patients with type I diabetes treated with continuous subcutaneous insulin infusion. Am J Med 82: 1127-1132

24. Lerman IG, Wolfsdorf JI (1988) Relationship of nocturnal hypoglycemia to daytime glycemia in IDDM. Diabetes Care 11: 636642

25. Whincup G, Milner RDG (1987) Prediction and management of nocturnal hypoglycaemia in diabetes. Arch Dis Child 62:333-337

26. Hirsch IB, Farkas-Hirsch R, Skyler JS (1990) Intensive insulin therapy for treatment of type I diabetes. Diabetes Care 13: 12651283

27. Wynne HA, Brown PM, Sönksen PM (1985) Acceptability and effectiveness of self-administered intramuscular insulin in juvenile-onset diabetes. Practical Diabetes 2: 32-33

Received: 11 January 1993 and in revised form: 22 March 1993

Dr. J.E.Henriksen

Odense University Hospital

Department of Endocrinology M

DK-5000 Odense C

Denmark 